\title{
Erratum: Approximate Solutions of the Cahn-Hilliard Equation via Corrections to the Mullins-Sekerka Motion
}

\author{
E.A. Carlen, M.C. Carvalho \& E. ORlandi
}

Communicated by T.-P. LIU

\section{Arch. Rational Mech. Anal.}

DOI: $10.1007 / \mathrm{s} 00205-005-0366-5$

Issue: Volume 178, Number 1

Date: October 2005

Pages: 1-55

In the online version of this article, the affiliation for one author was incorrect.

The correct information is given below

School of Mathematics, Georgia Institute of Technology, Atlanta, GA, 30332, U.S.A. e-mail: carlen@math.gatech.edu

and

School of Mathematics, Georgia Institute of Technology, Atlanta, GA, 30332, U.S.A.

on leave from Departamento de Matemática da Faculdade de Ciencias de Lisboa, 1700 Lisboa codex, Portugal. e-mail: mcarvalh@math.gatech.edu

$$
\text { and }
$$

Dipartimento di Matematica, Universitá degli Studi di Roma Tre, P. S. Murialdo, 1, 00146 Roma, Italy. e-mail: orlandi@mat.uniroma3.it 